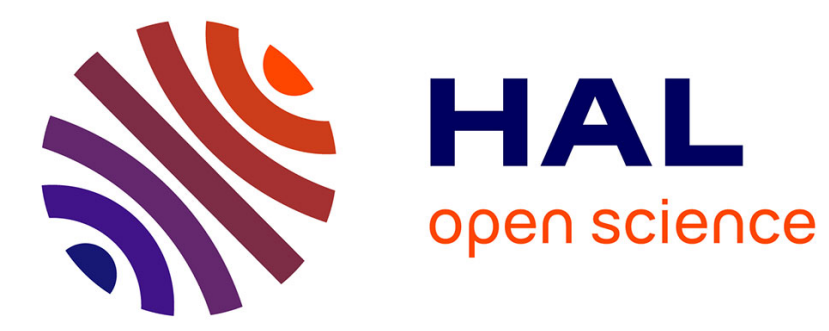

\title{
Deux moments dans l'histoire du Théorème d'algèbre de Ch. F. Sturm
}

Hourya Benis Sinaceur

\section{To cite this version:}

Hourya Benis Sinaceur. Deux moments dans l'histoire du Théorème d'algèbre de Ch. F. Sturm. Revue d'Histoire des Sciences, 1988, 41 (2), pp.99-132. 10.3406/rhs.1988.4093 . halshs-01119574

\section{HAL Id: halshs-01119574 \\ https://shs.hal.science/halshs-01119574}

Submitted on 23 Feb 2015

HAL is a multi-disciplinary open access archive for the deposit and dissemination of scientific research documents, whether they are published or not. The documents may come from teaching and research institutions in France or abroad, or from public or private research centers.
L'archive ouverte pluridisciplinaire HAL, est destinée au dépôt et à la diffusion de documents scientifiques de niveau recherche, publiés ou non, émanant des établissements d'enseignement et de recherche français ou étrangers, des laboratoires publics ou privés. 


\section{Deux moments dans l'histoire du théorème d'algèbre de Ch. F. Sturm*}

RESUME. - Au départ (1829), le théorème d'algèbre de Ch. F. Sturm fournit un algorithme pour compter le nombre de racines d'un polynôme sur un intervalle réel. Généralisé par A. Tarski (dans les années 1930), il devient un algorithme de décision pour la théorie logique du premier ordre du corps ordonne des nombres réels. L'article suivant considère ces deux moments : la naissance dı théorème de Sturm à partir du théorème analogue de J. Fourier ; sa transformation par Tarski qui en révèle la portée logique et inaugure ainsi le renouvellement auquel ce théorème doit encore son actualité.

Diverses questions générales sont abordées par le biais de cette étude : sur less échanges entre Algèbre et Analyse, sur les définitions par conditions nécessaires et suffisantes, sur la nature algébrique des méthodes, sur l'effectivité des prorésdures, sur la notion d'algorithme.

SUMMARY. - At the outset (1829), Ch. F. Sturm's theorem of algebra provided an algorithm for counting the number of roots of a polynomial which lie within an interval of real numbers. Generalized by A. Tarski during the 1930s, this theorem became a decision algorithm for the first-order theory in logic of the ordered field of the real numbers. In the following article, I consider these tw'o events : the origins of Sturm's theorem, which lay in J. Fourier's similar theorem, and the transformation of Sturm's theorem by Tarski, which displayed its logical significance and, in this way, ushered in the revival to which this theorem still owes its acluality.

From the siewpoint that I take in this study, I take up various general questions: about exchanging algebra and analysis : about definitions in terms of necessary and sufficient conditions; about the algebraic nature of methods; about the effectivity of procedures (meaning whateser enables procedures such as finitely recursise procedures to be carried out in a finite number of steps); and about the notion of algurithin.

- le texte est issu de deux conmunications, la premiere au lolloque d llistoire des Malhematiques, cırs (Marseille-Luminy, juin 1985), sur " lee théorème de Sturm $\cdot \mathrm{t}$ l'analyse des équations déterminees de .I. Fourier "; la deuxième au colloque international de P'hilosophie et d'llistoire des Mathématiques à l'Université autonome de Irexico décembre 1985), " Ilistoire et actualite du thiorime le Sturm ". Tel qu'il se présente ici, il constitue une étape dans lélaboration d'un travail plus ample, a paraitre en 1989, sous le titre: "Corps et modèles. Aspects de la construction de l'algèhre réelle. ".

Rev. Hist. Sci., 1988, XLI/2 
L'intérêt pour lo théorème d'algèbre do Ch. F. Sturm est lié a des questions telles qur : Qu'est-ce qu'un algorithme ? Qu'est-ce qu'unc méthode effective? Qu'est-ce qu'une propriété ou une procédure algébrique? Pourquoi la théorie des équations algébriques s'offre-t-elle comme paradigme pour la résolubilité d'un problèmı? Or, res questions, dont l'enjeu rst assez général pour tomber dans 1. champ d'unr épistémologie mathématiqur. sont posées avec assez d'rxactitude par la logique mathématique moderne. et en particulier par la théorie des modèles. Autant que la théorir de la démonstration, celle-ci a pris en effet le relais de la métamathémalique de Hilbert qui, à côté du problème du fondement des mathématiques (que nous mentionnons ici seulement pour mémoire), esquissait ou formulait des interrogations de ce type. A preuve la formulation dans les Grundzïge der theoretischen Logik (1928) du fameux problème de la décision qui a marqué toute une époque.

La théorir des modèles, au moins dans l'esprit des grands inifiateurs: Alfred Tarski et Abraham Robinson, prétend, par l'étude des langages de théories mathématiques particulières, prises justement pour modèles, obtenir dess schémas d'énoncés valables pour plusieurs théories a la fois ou, comme on dit, pour toute une classe de modèles. Une procédure, un théorème ne sont done pas étudiés pour "ux-mêmes, pour leur efficacité immédiate. Ils sont repensés dans le cadre d'une métathéorie, qui fait flèche aussi bien de l'information mathématique intrinsèque que de son interprétation logique pour $\mathrm{y}$ voir des modèles particuliers de srhémas plus généraux. Le gain étant que ces schémas généraux sont passibles d'une description formelle sachant exploiter les ressources d'un langage déterminé a priori par un vocabulaire de base et une grammaire.

Le philosophe sera naturellement sérluit par l'aspect formel de ce type d'analyse. Le mathématicien pourrait s'intéresser aux retombées techniques qui s'ensuivent le plus souvent. Car il n'est pas indifférent, du point de vue mathématique, de mieux délimiter le domaine de validité d'un énoncé mathématique, de s'apercevoir par exemple que celui-ci est beaucoup plus large que le domaine associé à l'origine et durant le temps d'une première évolution, forcément limitée par le domaine d'origine. Las résultats de la théorie des modèles peuvent ainsi constituer une source de renouvollement et de généralisation de résultats mathématiques.

Cet état de choses, qui milite en faveur d'une coopération entre 
mathématiques et logique, et done aussi en faveur d'une histoire qui tienne compte simult anément d'acquis de ces deux disciplines, est merveilleuseminl illust ré par le cas du théorème d'algèbre de Sturm.

I - ÉNONCÉ DU THÉORİME D'AIGËBRE DE STURM

Il s'agit du théorème présenté par Sturm à l'Aradémie des Srirnces le 2:5 mai 1829. énoncé et brièvement commenté dans le tome XI (juin 1829) du Bulletin de Férussac, dont Sturm était alors rédacteur. exposé complètement et démontré en 1835 dans l. "Mémoire sur la résolution des équations numériques" (1).

$C$ : théorèmr donne une méthode pour déterminer le nombre de racines réelles, comprises entre deux nombres réels $\mathrm{A}$ et $\mathrm{B}$. d'une iquation polynomiale $\mathrm{V}=0$ ou $\mathrm{V}$ a la forme :

$$
a_{n} x^{n}+a_{n-1} x^{n-1}+\ldots+a_{1} x+a_{0} .
$$

Cette méthodr consiste à calculer d'abord la dérivée $V^{\prime}$, puis it appliquer l'algorithme d'Euclide pour trouver lo p.g.r.d. (plus grand commun diviscur) de $V$ et $V^{\prime}$. On pose $V^{\prime}=V_{1}$, en sorte que $\mathbf{V}=\mathrm{V}_{1} Q_{1}+\mathrm{R}_{1}$. On dévie alors de l'emploi habituel de l'algorilhme d'Euclide 'n posant $V_{2}=-R_{1}$. La raison de ce changement de signe apparaîtra plus bas. On a donc $V=V_{1} Q_{1}-V_{2}$.

On réitère l'opération avec $V_{1}$ et $V_{2}$, pour obtenir :

$$
\mathrm{V}_{1}=\mathrm{V}_{2} Q_{2}-\mathrm{V}_{3} \text {. }
$$

On recommence avec $V_{2}$ et $V_{3}$ et on poursuit le processus de division juequ'à parvenir à un reste $V_{r}$ qui, si $V$ et $V_{1}$ n'ont pas de racine commune, cas auquel il est toujours possible de se ramener, est une constante numérique (l'indice $r$ étant majoré par le degré de $V$ ). On obtient ainsi 1. tableau :

$$
\begin{aligned}
& \mathrm{V}=\mathrm{V}_{1} \mathrm{Q}_{1}-\mathrm{V}_{2} \\
& \mathrm{~V}_{1}=\mathrm{V}_{2} \mathrm{Q}_{2}-\mathrm{V}_{3} \\
& \mathrm{~V}_{2}=\mathrm{V}_{3} \mathrm{Q}_{3}-\mathrm{V}_{4} \\
& \dot{\mathrm{V}_{r-3}} \cdot V_{r-2} \mathrm{Q}_{r-2} \cdot \mathrm{V}_{r-1} \\
& \mathrm{~V}_{r-2}=V_{r-1} Q_{r-1}-V_{r} .
\end{aligned}
$$

1) In Mémoires prespntes par divers savants a l'Acrudemie [royale] des Sciences de I Institut de Franre, 6 1835, $271-318$. Pour un aperçu de la vie et des travaux de Ch. F. Sturm, voir larticle de: P. Speziali dans le Jictionary of Scientifir Biography, XIII New York, 1976, 196-13?. 
La suite des fonctions polynomiales $\mathrm{V}, \mathrm{V}_{1}, \mathrm{~V}_{2}, \ldots, \mathrm{V}_{\text {r }}$ est communément appelée "suite de Sturm ", ou "chaine de Sturm " (l'usage de crette dernière 'xpression remontant à l'exposé du théorème de Sturm fait par Heinrich Webrer dans le tome I de son Lehrbuch der Algebra, §91-95) (2).

Etant donné deux nombres réels $\mathrm{A}, \mathrm{B}, \mathrm{A}<\mathrm{B}$, on écrit la suite $S_{A}$ des signes que prennent les fonctions $V, V_{1}, V_{2}, \ldots, V_{r}$, pour la valeur $A$; et de même la suite analogue $S_{B}$. On compte le nombre $N_{A}$ des variations de signe dans $S_{A}$, le nombre $N_{B}$ des variations dans $S_{B}$. On démontre que $N_{A}-N_{B}$ est exactement le nombre de racines réelles de $\mathrm{V}=0$ comprises entre $\Lambda$ et $\mathrm{B}$.

En son temps, ce théorème fit, à lui tout seul, davantage pour la célébrité de Sturm que tous ses travaux ultérieurs sur les équations différentielles du second ordre, dont l'intérêt ne ful perçu que par la suite (théorie de Sturm-Liouville, à son tour mieux connue aujourd'hui de la grande majorité des mathématiciens). Terquem, par exemple, dans un article des Nouvelles Annales de Mathématiques $\left(^{3}\right)$, écrit, en 1843 , que celte découverte représent. un "événement considérable ", et qu'elle: "produisit une profonde sensation... sur les géomètres de toute classe ". Il ajoute avec une pointe de malice que M. Nayer-Dalembert, " doué d'un grand esprit de spéculation et sachant le prix de toute "hose, acquit le droit de faire usage de (cette) invention "et l'inséra en 1832 dans le manuel qu'il publia avec M. Choquet, sous le titro Traité élémentaire d'algèbre. Choquet et Mayer-Dalembert furent rapidement imités par Lefébure de Fourcy $\left(^{4}\right)$ et par Bourdon $\left({ }^{5}\right)$; puis par A. Serret $\left(^{6}\right)$ et $H$. Weber pour ne citer an dernier que les deux auteurs de manuels d'algèbre les plus célèbres dans la deuxième moitié du xixe siècle. En fait. " exigé tacitement pour l'admission à l'école polytechnique ", comme le note encore Terquem, ce théorème était vite devenu "obligatoire pour tous les auteurs d'éléments ".

Du côté de la recherche, il ne resta pas non plus lettre morte. Il fut suivi, en effet, par toute une série de mémoires ou d'articles:

(2) Braunschweig : Vieweg und Sohn, 1892) ; trad. franc. (lu t. 1 :Paris: GauthierVillars, 1898).

(3) T. II (1843), 97-106; t. III (1844), 188-194; 2114-2113; 555-565; 577-580.

(4) Legons d'algèbre (Paris : Bachelier, 1833), chap. XIX, 4×3-493.

(5) Elémenls d'algèbre, $7^{\mathrm{e}}$ éd. Paris : Bachelier, 1834), chap. V'111, \$ III, 557-57\%.

(6) Cours d'algèbre superieure, 3 e éd. (Paris : Bachelier, 1\$66). 
visant à l'améliorer ou à le transformer, de la plume des plus grands mathématiciens comme Sylvester, Cayley, Hermite ou Kronecker, et aussi de moins grands comme Borchardt ou Darboux ( $\left.{ }^{7}\right)$. Il rngendra ainsi re que Sylvester a appelé "un cycle d'idées sturmiennes ".

(ie cycle s'arheva pourtant, sinon aver les travaux de Sylvester ${ }^{(8)}$ commr celui-ci voulait bien le croire, du moins avec la difficile théorie des caractéristiques de Kronecker (9).

Après la publication par Liouville des travaux de Galois et l'émergence, in algèbre, d'un mode de pensée structural et de plus en plus orienté vers la constitution de concepts abstraits définis par des systèmes d'axiomes, l'intérèt pour le théorème d'algebre de Sturm (et pour les questions d'analyse numérique) décrut suffisamment pour que celui-ci devint une matière facultative, reléguée dans l'oubli par les auteurs de manuels.

On peut nuancer; l'éclipse n'est pas totale. En Allemagne par :xemple, la notoriété et la fréquence d'utilisation du Lehrbuch der Algebra de H. Weber sont telles que le théorème d'algèbre de Sturm, qui s'y trouve exposé dans un chapitre de quarante pages, ne disparait pas de l'horizon des chercheurs et des professeurs. En particulier, Artin ef Schreier qui en font un usage important en 1926

?) On trouver: une liste de travaux procédant du theorème de Sturm dans (;. Mignosi, Teorema di Sturm e sue estensioni, Rendiconti del Circolo Matemalico di Pulermo, 49 (1925), 1-164; et une autre liste dans M. Lecat, L'intermédiaire des mathémaliciens, t. II, 2e serie (192:3), 44-47.

(8) Sylvester écril d'abord deux notes brives en 1839 et 1841 respectivement. lill 1853 , il consacre loute la quatrième partie de sa théorie des syzygies a des considérations inspirées par le théorème de $\mathrm{Sturm}$, avec des résultats récemment réactualisés par le développement de la théorie algébrique des formes quadratiques. Voir à ce sujet If. Benis-Sinaceur, communication au XVIle Congrès international d'IJistoire des Sciences (Berkeley, lalifornia, 31 juillet-8 aoùt 1985) ou l'ouvragr annonce au bas de la p. 99.

l.es travaux de sylvester auxquels nous renvoyons sont reproduits dans The Collected Mathematical Papers of James Joseph Sylvester (Cambridge, 1904), t. I, 45-46, 5:1-60), 396-398, 429-586, 609-619, 620-626, 627-629, 630-633, 634-636, 637-640.

(8) Flle est exposic dans plusieurs articles reproduits dans les Werke de Kronecker (I.eiprig, 1895-1930) : l. I (1895), 175-212, 213-226, 227-234 et 303-348; t. II (1897), $37-4(), 71-82$. Elle est résumée dans larticle rédigé par (C. Runge pour l'Enzyklopädie der Mathemalischen Wissenschaften mit Einschluss ihrer Anuendungen, 1 (1898-1904), 104-448; et présentée sommairement par H. Wetır, Trailé dalgèbre supérieure (Paris rituthier-Villars, $1 \times 9 \mathrm{si}, \$ 101$.

Un exposé moderne est domé par H. W. Siegber , Some historical remarks concerning degree theory. American Mathemalical Monlhly, 88 : 1981), 125-139. (Nous remercions M. F. Coste-IRoy pour cette référence.) 
dans "Algebraische Konstruktion reeller Körper" ( $\left.{ }^{10}\right)$ et A. Tarski qui le transforme (vers 1930) en méthode d'élimination des quantificateurs dans son mémoire sur la Complétude de l'algèbre el de. la géomélrie élémentaires (11), se réfèrent à l'exposé de H. Weber. Et le traité de B. L. Van der Waerden $\left({ }^{12}\right)$, qui consacre le dernier chapitre de son premier tome à la théorie d'Artin-Schreier. Io consigne naturellement.

Aux Etats-Unis, les mathématiciens sont, à la fin du XIx el au début $\mathrm{du} \mathrm{xx}^{\mathrm{e}}$ siècle, très liés à leurs maitres ou confrères européens, allemands en particulier. Certains manuels sur la théorie classique: des équations mettent le théorème de Sturm en bonne place. ('ést par exemple le cas du New First Course in the Theory of Equations de L. Dickson (13). Et l'histoire des mathématiques de Florian Cajori (1 re éd., 1893) relève à la fois le succès du théorème qui éclipse celui de $J$. Fourier, et l'étendue de son retentissement : en Angleterre (De Morgan, Sylvester), en Autriche (Andreas von Ettingshausen), en France (A.-L. Cauchy, J. Liouville, J.-..-(C. Duhamel, etc.) (14).

\footnotetext{
(10) Abhandlungen ans dem mathematischen Seminar dor I ninersitäl Hambury, : $(1926), 85-99$.

(11) Ce inémoire, prêt pour l'édition en 1940 (Paris : Hermann) ne parut qu'en 1967 sous le titre : The Completeness of Elementary Algebra und Goomelry (Paris : Institut Blaise Pascal) ; trad. franç. dans Tarski, Logique, semantique, melamathématique, $1 .: 2$ (Paris : A. Colin, 1974). Entre-temps, une deuxième version en fut publiée aux EtalsUnis sous le titre: A Decision Methol for Elemenlary .1lgebra and Reumelry iBerkeley: (:alifornia, 1948; 2e éd., 19:1).

(12) Moderne Algebra, 1 re id. Berlin, 1930). On peut remarquer, par contre, que Helmut Hasse, qui publie "ll 1927-1933 un petit manuel d'algèbre superieure (Sammlung Goschen, Bd. 931-932; Berlin : Walter de Gruyter mentionne dans une note (II, p. 79) la théorie d'Artin-Schreier; mais son livre, tout entier écrit dans l'esprit de la construction de l'algèbre abstraite, fait un exposé tolalement "modeme" de la théorie des équations en commençant par définir les corps, les anneaux, les groupes, les déterminants et en mettant à profit le mémoire de Steinit\% paru dans le .Journal de rirelle, 137 (1909). Dans cette perspective, il u'? a pas de place pour des considérations comme celle de Sturm.

(18) New York, 1932$), \S 57,58,59$.

(14) A. De Morgan a salué dans le théorème "la solution théorique complete d'une difficulté à laquelle s'rtaient employées des énergies de tout ordre depuis l'epoque de Descartes ". Vité par F. Cajori, A History of Mathematics New York : Macmillan, 18!3, 2e ed., 1919 i, 363.

Pour .J.-J. Sylvester, se reporter aux références de la $n . x$. Uonnons les références relatives aux autres auteurs :

... A. von Ettingshausen, Sturm's Regel... nebst einem Beweise derselben, $Z$. f. I'llys. Math. $u$. verwandte Wiss., t. $7,444-450$.

- A. J. Cauchy, Extrait du mémoire présenté à l'Académie de Turin le 11 octobre $1 \times 31$ (1832-1833) : Euvres, 2, XV, 262-411. - Extrait d'une lettre sur un mémoire
} 
Mais, en France, la première gloire du théorème de Sturm semble ternir. Sans vouloir établir une stricte chronologie de son maintien ou de son effacement, disons que l'extraordinaire développement en notro siècle de toutes les branches mathématiques fit de cet énoncé singulier un résultat marginal, rarement étudié ou mème simplement connu. Prenez, par exemple, dans les années 70-80, un bon étudiant ayant accompli à Paris le cursus normal d'une maìtrise de mathématiques: il a fait de l'algèbre, il connait un boul de la théorie des équations, il a même fait parfois de l'analysir numérique, sans jamais avoir entendu parler de la méthode de: Sturm. Il a sans doute consulté le manuel de R. Godement ou relui de Birkhoff-Mac Lane, il a feuilleté les fascicules d'algètre de Bourbaki ; nulle part il n'aurait pu trouver une mention, mème rapide, de notre théorème. Celui-ci ne semble plus faire partir du savoir vivant. Tout au moins du savoir académique, transmis par l'enseignement.

Les historiens dres mathématiques, dont les vues sur le passé dépendent beaucoup des résultats du present, le mentionnent tout aussi rarement. Le gros liver dr Morris Kline, Mathematical Thought from Ancient l" Modern Times (New York, 1972), qui s'est largement imposé avec une information couvrant deux millénaires et toutes les branches mathématiques, ne souffle mot du procédé de Sturn pour compler et localiser les racines réelles des polynômes. De fait, unc périodisation tranchée de la théorie des équations en epoque classique ou pré-galoisienne et époque moderne déterminée par Galois, Kronecker, Steinitz, ete., toute légitime qu'elle soit dans son principe, portr " naturellement. " à négliger certains résultats (dont le théorème de Sturm). A pru près contemporains, par leur date. de la théoric de Galois, reux-ci n'ont évidemment pas de raison d'apparaitro dans un chapitre sur "L'Algèbre du Xvıl e siècle "(p. ¿92-613 do M. Kline). Mais franchement différents par l'esprit, ils ne pruvent non plus figurer dans l'exposé de cette

publice : Turin le 16 juin 1833, Ch, 1. IV, p. 67: \& mai 18:37: ; CEutres, 1, I1, 45-48. Note sur un theorène relatif aux racines des equations simultanées, $r /, \mathrm{t} . \mathrm{V}$, p. 6 i3 juillet 18:37, ; Entres, 1, IN; 81-8:3. - Calcul des indices des fonctions,

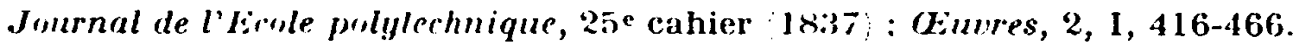

-- J. Liouville et Ch. Sturm, Démonstration d'un theorime de Cauchy, fonrnal de Limuville, 1 (1836), 27x-289. Et note publice aux $6: h, t$. IV, ler sem. (1837, 720-724. -... J.-M.-1. Duhamel, IC's méthodes dans les sciences de raisonnement Paris : GauthierVillars, 1867, 2e parlie, chap. XXVIII, no 231, 257-259. 
théorie, ni même dans une liste de résultats précurseurs. Les voilà donc promis à l'indifférence!

Indifférence encore de la part des rédacteurs bourbakistes des Eléments d'histoire des mathématiques (Paris, 1960). On ne peut, il est vrai, tout trouver dans des notices si condensées, mais seulement des résultats "très importants ", qui survivenl de façon ou d'autre dans les résultals actuels. Cependant, comment juger de l'importance des résultats actuels ou récents? Bourbaki, par exemple, s'est peu intéressé à la théorie d'Artin-Srhreier. Contrairement à $B$. L. Van der Waerden, il en donne un exposé très suecinct. où n'apparait pas le théorème qui utilise le résultat de Sturm (15). Aussi peut-il écrire, dans une de ses notices historiques, que la question de la séparation des racines d'un polynome est "un des sujets de prédilection de l'algèbre au xixe siècle "la taxant globalement d'une caducité qui rejaillit en particulier sur le théorème de Sturm $\left.{ }^{16}\right)$.

Ainsi, après avoir été porté aux nues par les autorités académiques de son époque, le théorème de Sturm se trouve aujourd'hui, ou jusqu'à une date récente, renvoyé par l'establishment mathématique au magasin des antiquités oubliées.

\section{II - STURM ET L'ANALYSh DES ÉguAtions déterminés DE J. FOURIER}

Cette situation donne un aperçu des caprices de l'histoire et de l'arbitraire des institutions autant que de la rationalité des évolutions.

D'une part, en effet, il est normal, quoique non obligatoire, qu'un résultat appartenant sans ambiguïté possible à la théorie

(15) Il s'agit du theorème d'unicité de la clóture rélle d'un corps ordonné. Voir 13. L. Van der Waerden, Moderne Algebra, I. La première édition du livre II, Algèbre de Bourbaki, chap. VI et VII (Paris : Jermann, 1952), mentionne ce théorème en renvoyant par une note (p. 38) aux pages utiles de la Moderne Algebru de 1 . I. Van der Waerden. La nouvelle edition (Algèbre, chap. 4 à 7 (Paris : Masson, 1981) supprime cette note au profit d'un exercice laissé au soin du lecteur : voir A.VI.24.

(16) Celui-ci n'a pas plus de chance dans le récent Abrégé d'hisloire des mathemaliques, publié en 2 volumes sous la direction de .J. Dieudonné Paris : Hermann, 1978; nouv. éd. 1986), mais l'Encyclopaedic liclionary of Mathematics publié par Iyanaga (éd. anglaise (Cambridge : . $1 \mathrm{~T}, 1977$ ) mentionne le thiorème de Sturm dans son article 296 (XV.5) B, t. II, 932 . 
classique des équations soit oblitéré par l'offensive de l'algèbre abstraite. D'autre part, le hasard déjoue bien des certitudes, nul n'ayant le moyen de prévoir a priori toutes les combinaisons possibles d'idées distinctes, ni, par conséquent, de juger définitivement de l'utilité ou de l'actualité d'un résultat donné.

L'algèbre abstraite se reconnait pour ancêtre Galois; pour précurseurs l'école formaliste allemande (Grassmann notamment) al l'école symbolique anglaise (Peacock, De Morgan, Boole), avec: une tradition maintenue vivante par Sylvester ef Cayley entre autres.

Or Sturm, qui édite certains articles de Galois dans le Bullelin de Férussac (entre 1829 et 1831), n'a pas le moindre soupçon du concept de groupe; ni a fortiori du concept de corps. L'idée d'opération abstraite est étrangère à l'ensemble de son ouvre, alor's que, à observer la stricte chronologie, elle est déjà présente dans les calculs de Gauss sur les nombres complexes ou sur les rlasses de formes quadratiques; et que la théorie va en être faite incessamment dans l'Ausdehnungslehre de Grassman (1844) ou les Lectures on quaternions de Hamilton (1853 - c'est l'année de la mort de Sturm !). Sturm fait d'ailleurs un usage tout à fait traditionnel des nombres réels sans attention aucune pour les règles formelles de leur calcul. Quant à son théorème d'algèbre, dérivé de se's travaux sur les équations différentielles (17), il ne met en œuvre que des éléments dont la combinaison inédite qu'il représente n'exige aucun élargissement du cadre de la théoric "classique " des équations : ni introduction d'un point de vue nouveau, ni modification, même partielle, du point de vue ancien. Pour ce qui concerne l'algèbre proprement dite, rien dans l'œuvre de Sturm n'annonce ni ne préfigure la mutation que va connaitre rette discipline à partir des années 1860 et jusque vers 1930 où le manuel de B. L. Van der Waerden, Moderne Algebra, apposera le sceau de la clarté didactique à une somme de recherches dont certaines remontaicnt au siècle précédent tandis que d'autres avairnt à peine trois ou quatre années d'existence !

Sturm fait connaitre son théorème an 1829 et le situe "xplicite-

(17) Cf. la première page de l'extrait de Sturn sur l'intégration d'un sýstème d'équations différentielles linéaires, Bulletin des sciénces malhemaliques, physiques el chimiques (Férussac), t. XII 1829), art. 196, 313-39: : et la remarque finale du mémoire Sur les equalions differentielles liniaires du second ordre, fommal do Jionuille, 1 (18:36), 186. 
ment dans le cadre de "l'analyse algébrique " telle que la comprenait J. Fourier. Il reconnait franchement sa dette envers son maître el ami. "Je déclare, écrit-il, que j'ai eu plrinc connaissance: de ceux des travaux inédits $\left({ }^{18}\right)$ de .I. Fourier qui se rapportent à la résolution des équations, et je saisis cette occasion de lui témoigner la reconnaissance dont ses bontés m'ont pénétré. " (19) Puis il ajoute : "C'est en m'appuyant sur les principes qu'il a posés, et en imitant ses démonstrations, que j’ai trouvé less nouveaux théorèmes que je vais énoncer. "

Compte tenu des autres déclarations de Sturm sur son thérème: d'algèbre $\left({ }^{20}\right)$, cette phrase résout deux problèmes. Le premier fut agité au Xıxe siècle et trouve un écho dans l'histoire des mathématiques de Florian Cajori (21). L'impression faite par le théoréme: de Sturm avait été si forte, en effet, que surgissait parfois la question de sa genèse ; on se demandait comment Sturm en était venu à cette " découverte capitale " $\left.{ }^{22}\right)$, à co " trait do génie " $\left.{ }^{23}\right)$. On oscillait entre l'idée qu'il s'agissait d'un " corollaire " des recherches mécaniques de Sturm $\left({ }^{24}\right)$, et celle d'une "illumination subite "( $\left.{ }^{25}\right)$ qu'il aurait eue à la lecture des papiers prêtés par Fourier. Les deux idées sont d'ailleurs émises d'abord par Sturm lui-même comme nous avons voulu le souligner. Et elles ne sont nullement incompatibles, car elles ne sont pas du même ordre. L'une renvoie? au contexte de la découverte : les travaux sur les équations différentielles, tandis que l'autre en détermine la lexture : la démons-

(18) Ces travaux de Fourier, inédits en 1829, seront rassemblés en un volune? publié par Navier sous le titre: Analyse des équalions délerminées Paris: F. Lidot, $18: 31\}$.

(19) Bulletin des Scienres mathemaliques, physiques of rhimiques Ferussac), 1. (1829), art. 271, 419-422.

(20) Voir les textes cités daus les 11.17 et 19 .

(21) Op. cil., in n. 14, p. :36:3.

(22) Liouville, Eloge funèbre de Sturm cité dans E. Prouhel, Notice sur la vie et les travaux de Ch. Sturm, in Ch. Sturm, Cours d'analyse de l'Erisle polytechnique Paris : Mallet-Bachelier, $1857-1859 ;$, t. I.

(23) Borchardt, Vouvelles Annales de Malheimatiques, t. 11 , $1452,403-406$.

(24) Liouville, Eloge funèbre de Sturm. Sturm a lui-mème textes cités dans lo's 11. 17 et 19) accrédité ce point de vue qui fut ensuite développé par M. Böcher dans un article souvent cité : The published and unpublished work of ih. Sturm on algebraic and differential equations, Bulletin of the American Muthematical Society, 18:1!111912), $1-18$.

(25) J.-M.-(:. Duhamel, Nouvelles Annales de Mathemaliques, 2e sirie, t. 6,1867 , 427-430 (Réponse à un compte rendu publié par Prouhet dans le même numéro : 234-240). I.'idée de Duhamel selon laquelle Sturm a trouvé son théorème par une a espèce de divination "n'est pas si éloignée que l'a pensé F. Cajuri de celle de Sylvester qu'il rapporte dans son ouvrage (p. 363). 
tration reprend celle du theorème de Fourier, en respecte les principes et en améliore les performances techniques. Aucune des deux no réduit complètement cr qui, dans une invention, échappe. à loute genèse psychologiqur.

Lautre problème fut posé assizz récemment dans lo contexte de l'exploitation logiro-algébrique lu théorème. Prompt. sans doute. a réinterpréter rétrospectivement un résultat en fonction de son utilisation acturlle, on s'est demandé si Sturm n'avait pas déjà eu idée dune démonstration purement algébrique, libre de toute considération infinitesimale, ou neme si, loutes proportions gardées, il n'avait pas un peu antiripé les exigeners finitistes du programme de Hilbert $\left({ }^{26}\right)$.

L'étude simultanée du "Mémoire sur la résolution des équations numériques " de Sturm et de l'Analyse des équations déterminées (1831) de Fourier montrera qu'il n'en ast rien.

\section{1 . Analyse algébrique et analyse infinitésimale selon Fonrier}

Le théorème principal de l'Analyse des équations déterminées est moins précis que celui de Sturm, puisqu'il donne une majoration du nombre des racines réelles d'un polynôme sur un intervalle, mais ne détermine pas ce nombre de façon exacte $\left({ }^{27}\right)$. Il s'agit, en fait, comme son auteur le souligne expressément, d'une généralisation de la règle de Descartes, qui majore le nombre des racines réelles positives d'une équation algébrique par le nombre de variations de signe dans la suit. des coeflicients de l'équation.

La lecture de l'ouvrage post hume de Fourier n'en ast pas moins

\footnotetext{
26: Voir par exemple G. Kreisel, What hatr we learnt from llitherts second problem?, Pror. "if Symprosin in Pure .Malh., vol. 28 :1976!, 111?.

(27) On identitle communement ce therrime de Fourier et le thereme analogue de Budan. Si cette identite est entiere, il faut renoncer a parler d" "thoreme de Budan-lourier car il rest facile de montrer lantérioriti des travinx de Fourier. Celui-ci etablit son theorime en 1796-1797 dans les lecons i l Ecole polytechnique; un résumi de ces lecons a ete conservi voir liverlissement de Navier it l'edition posthume de l'Anulyse des equalions delerminees. Mais on a remarqui recemment ques cetle identiflcation est abusive. 1 . G. A kritas, par exemple, pense que le thereme de Budan est "différent "quoique equivalent à celui de fourier. Voir, en particulier, Budun's Theorem and its conseguences, expose au Seminaire dillistoire des Mathimatiques de l'Universite de Nantes, publii daus sirmers of Tredmiques en perspertive, $\left.n^{\circ} 4: 1384\right), 1-13$.
} 
instructive. Ne serait-ce que par l'énonciation explicite des principes que Sturm reconnait avoir suivis.

Qu'est-ce que Fourier entend par "analyse algébrique "?

Cette expression. usuelle depuis lat fin du xvine siècle, reçoit dans l'ouvrage de Fourier deux définitions, l'une par extension, l'autre par compréhension. La première se réduit à énumérer en respectant la chronologie (Préface. p. 2) un certain nombre d'éléments dr la tradition mathematique:

- Les théorèmes de Viètr sur la composition des coeffirients,

- la règle de Descartes,

- la règle du parallélogramme de Newton,

-.. la méthode newlonienne des substilutions successives,

- les recherches de Waring et de Lagrange sur les fonctions invariables des racines et sur l'équation aux différences,

- La théorie des fractions continues telle qu'elle est expliqué dans les ouvrages de Lagrange $\left({ }^{28}\right)$,

-. la méthode de D. Bernoulli, déduit des séries récurrentes.

Il s'agit, comme on le voit, d'éléments de la théorie classique: des équations, avec en bonne place l'aspect formel et combinatoire des recherches sur les fonctions symétriques des racines.

La seconde définition, qu'on ne trouve pas loute faite dans l'Analyse des équations déterminées, est cependant imposée par la philosophie mathématique générale de Fourier et ses idées particulières sur la solution des problèmes laissés en suspens par la résolution algébrique des équations.

En premier lieu, Fourier pense que "les connaissances mathématiques les plus diverses sont toutes de même nature "; qu'il n'y a done pas de séparation nette entre les questions dites d'algèbre et les questions diles d'analyse. Grosso modo, l'algèbre était alors représentée par la théorie des equations et l'analyse par le calcul infinitésimal. Mais Fourier, pour qui le terme "analyse" a d'ailleurs une résonance qui outrepasse les limites du domainc strictement mathématique $\left({ }^{29}\right)$, appelle "analyse générale " la science des prin-

\footnotetext{
28) Fourier songe probablement aux Xotes et Additions de lagrange il la traduction francaise (par Jean II Bernoulli) (Lyon, 1774), des Eléments d'Algebre d'Euler. Ces additions comportent, en effet, un exposé de la théorie des fractions continues.

(29) "L'objet de l'Analyse, écrit-il, est un élement préexistaut de l'ordre universel. . Ou encore " L'étude approfondie de la nature est... un moyen assure de former l'Analyse
} 
cipes qui gouvernent toutes les mathématiques et donc aussi bien la theorie des equations (lalgèbre) que le calcul infinitésimal (l'analyse). Dans cette oplique unificatrice, il y a une étroite coopération entre l'analyse algébrique et l'analyse infinitesimale.

D'une part en effet, "les questions les plus importantes de la philosophir naturelle, comme celles qui ont pour objet d'exprimer les dernières oscillations des corps, ou la stabilité du système solaire, ou divers mouvements des fluides, ou enfin les lois mathématiques de la chalrur, exigent une connaissance approfondie des équations" $\left.{ }^{30}\right)$. D'autre part, "on ne preut faire aucun progrès considérable dans la theorio des équations sans quelque usage de l'analyse differentiolle, ou, ce qui est la mème chose, de la thérie des fluxions " Préface, p. 3-4). Fourier sait dans quelles difficultés s'empêtrait, au début du xixe siècle, la résolution algébrique des équations et mesure l'intérèt at l'utilité d'unr résolution numériqur. Dans l'introduction à son ouvrage, il passe en revue et caractérise succinctement tous les travaux connus sur les équations, de Léonard de Pise a Lagrange avec, entre les deux, Cardan, Vièle, Wallis, Descartes, Newton, Albert Girard, De Gua, Rolle, Leibniz, Tschirnhaus, Vandermonde et j'en passe! si la résolution algébrique fait problème, la résolution numériqur "doil ètre regardée comme une des plus importantes applicalions $\left({ }^{31}\right)$ du ralcul différentiel " (Introduction, p. 8, 18; exposé synoptique, p. 84-85). Aussi, le lecteur est-il averti dr la nécessité de connaitro et «les principes » et "l'usage» de cre dernier $\left({ }^{32}\right)$.

Pour des raisons de symétrie aut ant que par souri d'exactitude, il faut aussi noter le rôle de ce que Fourier appelle "l'analyse des courbes", r'est-à-dire les ronsidérations géométriques sur les langentes ou sous-tangentes en un point qui correspond à une rarine de la fonclion polynomiale donnée. L'analyse des rourbes concourt, de façon adjuvante ou heuristique, à la solution des problemes qui ressortissent, tant par leur nature que par la méthode de

alle-mène, et d'en dicouvrir les alintents qüil nous importe de plus de connailri..." Thenrie analylique de le rhaleur, Discours preliminaire, in Genres, I, p. XXI). Et plus loin : "... l'Analỵse suit la même marche dans l'étude de tous les phénomènes; elle les interprite par lo mène langage, comme pour attester l'unite et la simplicite du plan de l'univers, et rendre encore plus inanifeste cot ordre immuatile qui priside a toutes les calises naturelles.

30 Souligné par nous.

81: Souligné par nous.

32. Lnalyse des équalions determinses, p. 18. 
résolution, au calcul algébrique ct à l'analyse infinitésimale simultanément. En effet, les relations exprimées par les équations

" n'appartiennent pas seulement à la science abstraite du calcul : elles r.xistent ${ }^{{ }^{33}}$ ) dans les propriétés des courbes et des surfaces, dans les mouvernents des solides et des fluides. dans la distribution de la chaleur. et dans la plupart des phénomènes naturels "( $\left(^{34}\right)$.

La forme dr la rourbe, déterminér au moyen du calcul des dérivées successives permet en retour de "connaître distinctement. la nature de la fonction primitive ". d'où l'on déduit enfin la répartition des racines de l'équation donnér. Ainsi les constructions géométriques tantòt rendent " très sensibles " les pésultats du ralcul, tantôt aussi permettent de les prévoir ( $\left.{ }^{35}\right)$.

Si la résolution d'une équation dépend do lapplication de la théorie des fonctions telle qu'elle s'est constitué à partir du xvire siècle, ainsi que des techniques du ralcul intégral et différenticl, c'est qu'on présuppose toujours que la fonction correspondant à l'équation - qui, du reste, peut être algébrique ou transcendante - est continue. Sans l'hypothèse de continuité, au moins lorale ou par morceaux, toutes les formules de l'analyse infinitésimale seraient dénuées de sens.

Il y a une correspondance parfaite entre l'application des principes de l'analyse algébrique et l'application des principes do l'analyse infinitésimale. Car, si la première est fondée, comme l'écrit explicitement, Fourier $\left.{ }^{(36}\right)$, "sur la notion générale des variations -l des permanences de signe" (instituée par Descartes), on ne peut supposer, par aillrurs. que "les valeurs passent du positif au négatif sans devenir nulles dans l'intervalle "( $\left.{ }^{37}\right)$. C'est-à-dir qu'appliquer la notion générale des variations at des permanences de signe ne permet de conclure que dans le cas d'une "variation par degrés insensibles " ou continue. "Et lorsque cette condition n'a pas lieu, précise Fourier, rien n'empêche d'examiner séparé-

(33) Souligné par nous.

(34) Ibid., p. 21 . Voir les remarques en ce sens dans les voies de l'analyse classique, rililique, no $327-328$;aout-septembre 1974 ;.

(35) Par exemple, sur un intervalle où les racines de $f(x)=0$ sont en nombre pair, la construction des tangentes et sous-tangentes it la courbe associé a $f(x)$ guide la défnition d'un critère numérique pour savoir si les racines sont réplles ou innaginaires. Voir Analyse des équations delerminées, p. 117 sq.

$\left.{ }^{36}\right)$ Ibid., exposi synoptique, p. 65.

(37) Ibid., p. 59-60). 
ment chacune des parties où la continuité subsiste ". L'hypothèse de continuité est donc une condition sine qua non de l'analyse des équations numériques. Elle fait basculer la résolution du cadre algébrique, où prime lo point de vue équalionnel, à celui de la théorie des fonctions. Il était alors bien naturel que Fourier aùt l'idée de substituer, dans l'application de la règle des signes, la suite des fonctions differentielles d'une fonction donnée à la suite des coefficients de l'expression exprimant cette fonction. Cette appliration nouvelle resitur la règle de Descartes dans un contexte différent de son contexte d'origine.

Sturm, ayant déclaré sa fidélité aux principes de Fourier, n'a pas besoin de revenir explicitement sur l'hypothèse de continuite. Ellr est acquise. elle va de soi. Comment supposer alors qu'il ait voulu l'éviter? On peul dire, au contraire, qu'elle a pour lui un degré d'évidence tol qu'il n'a pas, de la première pagr de son mémoire à la dernière. l'ombre de la moindre réserve.

\section{:L'impéralif d'effectivité}

Biten que Fourier connaisse toutes les recherches anterieures $\therefore$ IIr la théorie des équations, c'est essentiellement par rapport à Lagrange (Traité sur la résolution des équations numériques de tous les degrés, 1798) qu'il se définit.

Fourier mesure parfaitement l'enjeu d'une résolution algébrique des équations. Celle-ci consiste:

"i assigner pour une equation proposée d'un degré quelconque un nombre limité d'opérations, tellement ordonnées que le résultat de la derniere fât une des racines, en n'admettant au nombre de ces opérations à eflectuer que les règles élémentaires du calcul $(-\ldots, \ldots,-)$ et celles qui sont indiquées par les signes radicaux" ${ }^{(38)}$.

Comme tout malhématicien intéressé par la théorie des équations, Fourier sait le prix d'un algorithme de résolution générale, qu'il décrit comme une "opération régulière et facile dont la marche "st. toujours la même" $\left({ }^{39}\right)$. Nalheureusement, "l'analogie du second dregré est trop incomplète "en ce qui concerne les degrés supérieurs.

\footnotetext{
38: Itid., p. 13, mais cest nous qui soulignons la périphrase par laquelle fourier vise la notion dalgorithme.

39) Ibid., p. 16.
} 
Fourier sait l'impossibilité de la résolution algébrique générale. Sans s'arrêter à une démonstration " régulière ", il en souligne. l'inutilité prévisible (40) ot insiste sur l'utilité d'une résolution numérique.

Celle-ci a dailleurs l'avantage de fournir non seulement un procédé de résolution mais aussi des solutions effertives, du moins si le procédé est d'application facile. Celui de Lagrange, par rxemple, l'équation aux carrés des différences, irréprochable théoriqurment, est impraticable ( $\left.{ }^{41}\right)$ ! Alors que les difficultés attachées a une résolution algébrique générale conduisaient à dissocier deux problèmes : celui, prioritaire, de l'existence d'une solution par radicaux, et celui du calcul de cette solution quand clle existe $\left({ }^{42}\right)$, Fourier maintient indissociées existence de principe et existence effective. Par exemple, même les formules connues pour la résolution algébrique des équations du troisième et du quatrième degré le laissent insatisfait. Car elles comportent des calculs trop complexes pour conduire rapidement à "la connaissance effective des racines"; elles s'écartent $d u$ " but réel " qui est de "connaitre en nombres, ou en une suite de monômes " ces racines.

(c) parti pris évident de calculabilité, Fourier l'a constamment

40; Ibid., p. 15. Rappelons que le fameux mémoirc d'Abel sur l'impossibilité de résoudre algébriquement les equations d'un degré supérieur au quatrième fut republié dans le premier volume du .Journal de Cirelle, en 18?6. I.a même année, un compte rendu en est donné dans le Bullelin de Férussac (t. 6, $1^{\circ} 207,347-354$ ), avec une note sur les résultats antérieurs de Rumini et le rapport favorable qu'en fit cauchy devant l'Académie des Sciences. A posteriori, on peut naturellement miditer sur la signification de l'adjectif "rigulière" sous la plume de Fourier!

(41) En ce qui concerne la résolution numérique, Fourier fait le point de la façon suivante (ibid., art. 35 du liv. II) : "La difficulte de distinguer le cas des deux racines imaginaires du cas des deux racines réelles est le point le plus important de l'analyse des squations; elle exige une méthode propre fondé sur le calcul des limites rntre lesquelles les racines sont comprises. Les recherches de Holle, celles de De Grua, n'ont pu conduire à la résolution numérique des equalions, parce qu'elles manquaient d'un caractère spécial pour distinguer les racines imaginaires. I. calcul de l'équation aux carrés des différences a résolu pour la première fois cette singulière difficulté; mais comme on l'a remarqué depuis longtemps, la solution est purement théorique, et les tentatives que l'on a faites pour la perfectionner... ont été presque entièrement infructueuses. Il itait donc nécessaire de traiter la question d'une manière différente... non moins exacte et d'une application incomparablement plus facile. "Sturm a repris le jugement de Fourier sur Lagrange ; voir le premier paragraphe de son mómoiro de 18:35.

(42) Lagrange, par exemple, en distinguant nettement la résolution algélrique des autres types de résolution, conçoit que la premiłre n'obéisse pas forcément a l'impératif deffectivité. Voir son introduction du Traité de la résolulion des équations numériques de lous les regrés, p. 16 de l'edition Serret (1879). 
défendu dans sa pratique de l'analyse différentielle (résolution de l'équation différentielle correspondant à la propagation de la chaleur). L'évolution, au xixe siècle, de la théorie des équations différentielles qui devient (aver Sturm précisément, puis Liouville) une théoric abstraite, davantage orientée vers la détermination theorique de lexistence et de la forme des solutions intégrales que vers le calcul d'intégrales particulières. l'évolution de la théorie des fonctions qui formalise ses concepts fondateurs, l'évolution de l'algèbre anfin qui aristallise ses procédés en concepts plutôt que de les développer en algorithmes, tout cela concourt à voiler provisoirement l'intérêl théorique des méthodes effectives. Kronecker, notamment influencé par le théorème de Sturm qu'il connaît bien, allait certes so faire le grand champion des méthodes effectives. Mais ses justifications auront une allure métaphysique. C'est Hilbert. qui en posant le problème de la décidabilité, révélera la dimension métamathématique de ces méthodes. Le développement de la théorie des fonctions récursives, d'une part, la mise au point d'algorithmes de décision pour des théories mathématiques ou logiques particulières, de l'autre, restitueront sur un autre plan le parti pris de calculabilité. En transformant le théorème de Sturm en algorithme de décision pour l'algèbre et la géométrir élémentaires, Tarski se basera sur son caractère effectif, propriété exigée explicitement par les principes de Fourier que Sturm a faits siens.

III - LA DÉMONSTRATION DE STLPM

\section{1 / Les principes de Fonrier}

En adoptant les principes de Fourier, Sturm combine naturellement calcul algébrique et analyse infinitésimale dans la démonstration de son théorème. Son mémoire de 1835 dresse un argument où l'on peut distinguer des hypothèses générales, qui en délimitent le cadre. et des méthodes plus particulières, qui en déterminont la spécificité. Font partie des présupposés généraux l'hypothèse de conlinuité et la méthode d'inspection des variations et des permanences dr signe. L'une ressortit, on l'a vu, à l'analyse infinitésimalr. l'autr" au calcul algébrique ; les deux étaient déjà employées par Fourior. Les méthodes spécifiques sont l'algorithme 
d'Euclide appliqué, de faģon modifiée (43), aux polynômes; ir théorème des valeurs intermédiaires selon lequel toute fonction continue qui change de signe sur un intervalle réel s'annule nécessairement entre les bornes de cet intervalle (aver une réciproque du style : toute fonction continue qui s'annule pour une valeur réclle $x$ qui n'annule pas sa dérivée change de signe sur un intervalle contenant $x$ ), et le thérème de Taylor. Ces trois derniers théorèmes, qui présupposent la notion de continuité, sont évidremment. dans la forme où ils apparaissent tant che\% Fourier qur chez Sturm, du ressort del'analyse in finitésimale. L'algorithme d'Eurlide, qui, appliqué aux polynomes, est souvent appelé par les auteurs allemands algorithme d'Euler, y échappe. Or, il constitue dans lo, théorème de Sturm. le seul élément absolument nouveau par rapport au théorème de Fourier.

Cette dissection de la démonstration du théorème d'algèbre de Sturm illustre assez la fidélité aux principes de Fourier qui prône, dans un esprit anti-lagrangien, le recours aux méthodes dr l'analyse différentielle.

Une analyse détailléo du mémoire de Sturm, qui ne saurait ètre menée dans les limites de cet exposé, montrerait la complémentarité exacte des arguments algébriques et des arguments infinitésimaux, avec, pour ces derniers, une préémin‘nce due à ce que tous les raisonnements présupposent l'hypothèse de continuité. En fail, il ne suffit pas de démèler, dans la démonstration de Sturm, les arguments algébriques d'avec les arguments infinitésimaux, il faut encore voir comment ils se ronjuguent et par quoi passe, en définitive, leur efficarité.

Prenons la méthode d'inspection des variations ou des permanences de signe. Elle est, depuis IDescartes au moins, d'un large usage dans toutes les questions relatives aux racines réelles des équations. Comme elle passe pour etre un outil algébrique, elle parait irréprochable du point de vue de ceux qui répugnent aux notions infinitésimales. L'étude simullanée de son emploi par Lagrange, Fourier et Sturm montre, cependant, que son statut n'est, en fait, pas si univoque qu'il le paratt. Statut et mode d'emploi sont liés. Voyons de plus près.

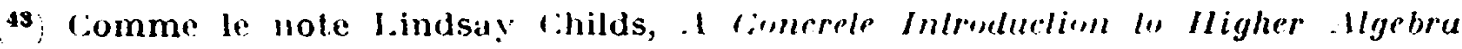
New York-Berlin : Springer-Verlag, 1979 ; chap. 7 , il s'agit d'une "application intelligente de l'algorithme d'Euclide ". 
Une des formes d'utilisation principales de cette méthode apparait dans la corrélation entre racine réelle et changement de signe d'une éfuation. Or, cette corrélation peut èt registres mathématiques divers.

Lagrange s'en tient. pour sa part, à une version purement algébrique, représentér par les théorèmes I et II du chapitre I de son Trailé de la résolution des équations numériques (1798) (44). Dans la démonstration de "es deux théorèmes "qui sont la base de loute la théorie des equations ", Lagrange veut éviter le recours à " la théorire des lignes courbes "et s'en tenir à "la nature mème " des équalions. Cependant il s'y reprend à deux fois (chap. I et n. 1) sans parvenir à un résultat satisfaisant.

Après cet échec, Fourier, on l'a dit, opte franchement pour le recours à " la théorie des lignes courbes ", rt justifie ce recours par les principes que nous avons évoqués.

Sturm, à son tour, fonde son analyse des équations différentielles autant que son théorème d'algèbre sur ladite corrélation en lui donnant la forme suivante.

D'une part, il admet comme vérité évident a que toute fonction continue, qui change de signe entre deux nombres récls $A$ et $B$. s'annule au moins une fois dans l'intervalle (A. B) (45). D'autre part et réciproquement, il énonce et démontre non on toute généralité mais dans le contexte de son argumentation, que si une fonction continue s'annule pour une valeur a qui n'annule pas sa

44) Rappelons le texte dr: I.agrange : "Theorime I : Si l'on a une équation quelconque, et que l'on connasse deux nombres tels qu'etant substitues successivement a la place de l'inconnue de cette equation, ils donnent des risultats de signes contraires, l'équation aura nécessairement all moins me racine rélle dont la valeur sera entre ces deux nombres.

"Théoreme II : Si dans unt iqualion quelconque, qui a une oll plusieurs racines réelles et inrgales, on substilue successivement a la place de l'incomme deux nombres dont l'un soit plus grand et l'autre soit plus petit que l'une de ces racines et qui diffirent. en mème temps l'un de l'autre d'une quantile moindre que la diffórence entre cette macine el chacune des antres racines réclles de l'équation, ces deux substilutions donneront nécessairement deux résultats de signes contraires. "

45: Mémoire sur les equations différentielles lincaires du second ordre, $\$$ XIV, Iournal de Liouville, 1 1836;, 132 ; Memoire sur une classe d'éfuations a différences partielles, $\$ \mathrm{XXV}$, ibid., 132 ; Mrmoire sur la résolution des éfuations numériques,

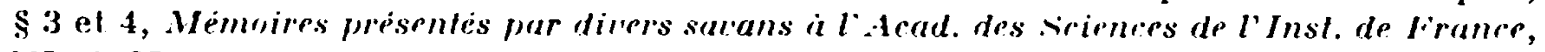
II 1835 ).

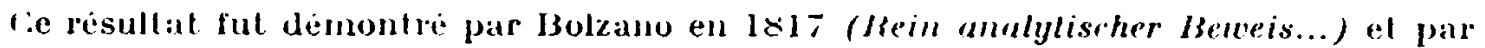
(auchy en $1 \times 21$ (Cisurs diunalyse algebrique). 
dérivée, alors elle change de signe sur un intervalle contenant $\alpha\left({ }^{46}\right)$.

Il ne s'agit plus d'équation, mais de fonction, et de fonction continue ainsi que de dérivée. De Lagrange à Sturm la formulation a totalement changé; les concepts et donc les méthodes sont tout autres. Comme chez Fourier, l'inspection des variations ou permanences de signe se trouve donc: immergée dans un contexte qui emprunte beaucoup a la théorie des fonctions et au calcul infinitésimal. Son efficacité présuppose de façon essentielle le concept de continuité.

Sturm ne présente ni ne discute explicitement les réquisits de son argumentation comme nous venons de les schématiser. Cependant il est impossible de ne pas les y voir, pourvu qu'on lise le texte de son mémoire. Du reste, ne s'agit-il pas là de présupposés très généraux dans le contexte de l'analyse algébrique du début du $\mathrm{XIX}^{\mathrm{e}}$ siècle (47) ? Et ces présupposés n'ont-ils pas été énoncés par Fourier sous la forme explicite de principes, auxquels Sturm a déclaré son adhésion?

\section{2/L'esprit d'abstraclion de Sturm}

On peut faire. rependant, unc lecture plus distanciée du textr. de Sturm. Après tout. Le succès d'un résultat se mesure souvent à l'écart entre les idées qu'il inspirr et celles dont il est issu !

Or. il y a une sorte d'effet "objectif " du texte de Sturm, produit à moitié par des qualités intrinsèques et à moitié par une projection rétrospective, qui en majore l'aspect algébrique au détriment de l'aspect analytique.

L'idée d'éviter less arguments géométrico-analytiques de continuité n'est ni explicitement défendue ni implicitement soutenuc par Sturm. D'ailleurs, c'est seulement à la fin du xixe siècle qu'elle devient une des idées mathémaliques dominantes (Dedekind, Hilbert en particulier). Ce qui est vrai c'est qu'il y a, en dépit de l'identité des principes. une différence générale de style entre Fourier

46; Sur les fqualions difrentielles lincidres, $\$$ II ; Ménoire sur la resolution des equations numeriques, $\$ 4$.

47) Voir Je: Contrs d'-Anulyse algébrigue de Canchy et le rinurs d'slgèbre superieure de A. Serret. 
et Sturm, et une différence plus particulière au théorème d'algèbre, qui facilite sa récupération a posleriori par les amateurs de méthodes algébriques.

a Nous n’insisterons iri qu'en passant sur le style général du travail de Sturm grâce auquel on doit la première théorie abstraite des équations différentielles. Quelle que soit la nature de l'équation. différentielle ou algébrique, Sturm attaque le problème de sa résolution en cherchant à déterminer le nombre de changements de signes sur un intervalle réel de rertaines fonctions. Cette méthode, il ne l'emploie pas seulement dans le cas où lo calcul direct des racines (rérlles). ast difficile ou impossible. Pour lui, rlle constitue de loutr façon un préalable. Car elle pernet de déceler a priori les propriétés, sinon les valeurs, des fonctions intègrales qu'on ne sait pas calculer. Comme dit Sturm, elle donne sinon la solution effective, du inoins "la forme " de la solution, c'est-ád-dire l'ensemble des propriétés prévisibles à partir d'une analyse de la forme de l'équation proposée.

Sturm se détourne ainsi des développements en sinus ct rosinus d'ares multiples de Fourier et tàhe de "reconnaître les propriétés caractéristiques " des fonctions et les sinuosités des courbes intégrales sur "la seule considération des équations différentielles" (48). Ce faisant, il applique à la résolution des équations différentielle's une idée caractéristique de l'algèbre traditionnelle des polynômes : le nombre et la nature des racines fournit une caractérisation antéricure à tout calcul effectif. C'est ce lransfert de méthode qui produil la mutation bien connue dans l'histoire des équations différentielles et représentér par ce qui ast appelé la "théorie de SturmLiouville ".

Gette mutation relère, chrz Sturm, de la mème disposition que relle par laquelle on va le voir définir les "suites de Sturm ". Elle exprime également la conviction - alors peu banale -- qu'il est possible d'obtenir des énoncés mathématiques intéressants sur des objets numériquement indéterminés. Et qu'il est sourent plus facile et plus rentable de saisir globalement des ensembles plutòt, que des éléments particuliers : la forme de l'ensemble des solutions d'une équation différentielle donne, à moindres frais, plus d'infor-

\footnotetext{
48: Voir, par exemple, lintrobluction du " Mrmoire sur les cirations différentielles linéaires du second ordre ${ }^{\circ}$
} 
mation que le calcul d'une ou plusieurs solutions particulières; de mème, la forme d'une "suite de Sturm "va résoudre un problème sur lequel les mathématiciens bulaient depuis longtemps.

$b /$ C'était en effret, comme le souligne Fourier à tant de reprises ( $\left.{ }^{49}\right)$, une question bien difficile que celle de la nature, réclle ou imaginaire, des racines d'une équation algébrique.

Rappelons, par "xemple: que la règle de Descartes ne nous dit pas s'il y a des racines réclles, mais seulement, au cas où il $y$ en a, que leur nombre ne peut dépasser celui des variations de signe de la suite des coefficients de l'équation proposée. Lagrange, qui avait théoriquement résolu le problème par la formation de l'équation dont les rarines sont les rarrés des différences entre les racines de la proposée, doit cependant se restrcindre au cinquième degré ou faire une hypothèse particulière sur le nombre des racines imaginaires $\left.{ }^{50}\right)$. Fourier a l'idé do combiner la règle de Descartes avec le théorème de Rolle en dénombrant les variations de signe de la suite des dérivées successives de la proposée. Mais, comme Descartes, il n'obtient qu'un majorant du nombre de racines réelles, à moins que l'équalion considérée n'ait toutes ses racines réclles, et le risque n'est pas supprimé de chercher des racines dans des intervalles où il n'y en a pas.

Connaître le nombre exact de racines réelles d'une équation algébrique. c'est connaître du même coup le nombre de ses racines imaginaires, et le problème de la nature des raciness se trouve alors résolu. Sturm at frappé par "l'incertitude " dans laquelle nous laisse mème le théorème de Fourier, comme il ressort des premières lignes des son mémoire sur la résolution des équations numériques. On peut penser que, ayant eu l'occasion "d'étudicr à loisir " les papiers personnels de Fourier sur la question, il a dû remarquer aussi la longucur et la multiplicité des procédés de celui-ci (51). Dans le cas général en effet, le théorème de Fourier doit obligatoirement être assorti de règles complémentaires si nombreuses qu'on se trouve pris dans les entrelacs d'une sinueuse analyse de cas. La

\footnotetext{
49, Analyse des équalions lislerminess, Introd. p. 11), 35 al.; Ixpose synoplique, p. 2!, 2e al.; liv. II, art. 3.;.

(50; Traile de la ressolulion des équalions numériques de lous les degrés, chap. I, art. II, no 36 .

51) Analyse des équations delerminées, $\mathrm{p} .31$ is 33 ; ou liv. $1, \$ 20$ à 38 . Le $\$ 38$, qui st un risume des diffirents cas ou etapes, couvre à lui seul trois pages el demie.
} 
méthode d'inspection des variations de signe ne peut ètre appliquée de façon uniforme, ni surlout univoque.

La principale entrave à cela est. comme l'a remarqué Fourier lui-même, que la diminution du nombre de variations de signe ne correspond pas forcément à une racine réclle de l'équation $f(x)=0$. La situation est ambiguë ; sauf dans un seul cas, celui d'une fonction telle qur si $f(a)=f^{(i)}(a)=0$ pour un certain nombre réel $a$, alors $f^{(i-1)}(a)$ et $f^{(i+1)}(a)$ sont de signes opposés. La particularité de ce cas s'oppose à un raisonnement général.

Que fait sturm devant cet obstacle majeur? Par un artifice. dont on peut trouver d'autres "xemples dans l'histoire des mathématiques il transforme l'obstacle en levier. Du ras isolé il fail un cas typique. C'est-à-dire qu'il prend la condition restrictive justement comme critère de définition, et pose a priori que les fonctions, auxquelles s'appliquera (de facon vraiment efficace parce que univoque) la méthode d'inspection des signes, devront satisfaire la condition isolée par Fourier dans un cas particulier. A celle-ci seront associées trois autres conditions mais d'importance moindre $\left({ }^{52}\right)$. Si bien qu'on arrive à une définition de type axiomatique (avant la lettre) qui ne désigne pas un objet particulier, telle suite particulière de fonctions, mais détermine un ensemble de suites (les "suites do Sturm ") par la donnér a priori d'un petit nombre de propriétés caractéristiques. Sturm est parfaitement conscient du caractère de sa démarche. Il expose d'abord complètrment sa démonstration sur le mode naif : il construit explicitement une " suite de Sturm " particulière et montre qu'elle fait l'affaire. Puis, sur un mode plus savant (plus abstrait), il dégage, ct énumère les propriétés nécessaires et suffisantes qui définissent des suites du inêmr type, te souligne ainsi que la conjonction de res propriétés détermine non pas une suite unique mais une infinité.

Sturm nous donne ainsi un très bel exemple du processus par lrquel une définition abstraite est forgér dans l'étude d'un cas particulier. En tranchant dans le vif, en se débarrassant d'entrée de jeu de tous les cas ambigus pour ne s'interesser qu'au seul cas uniroque, en se donnant artificiellement les conditions qui gouvernent ce cas, son théorème atteint une clarté, une simplicité

32; Non s, lo et l I du minoire de Sturm. Pour une formulalion générale de ces conditions, voir l'expose de II. Weber. 
et une généralité qui manquaient à celui de Fourier. Grâce à lui, la méthode d'inspection des variations ou permanences de signe, à laquelle Fourier avait donné une portée bien plus générale: qu'elle n'avait chez Descartes, Rolle ou Lagrange, devient enfin un outil sûr : rlle fonctionne dans des conditions telles que toute diminution des variations de signe correspond exactement à l'existence d'une racine réelle de l'équation proposér. On n'a plus besoin alors de la "considération des lignes courbes ", ni, en particulier, de l'étude. indispensable pour Fourier ${ }^{(53)}$, du rapport numérique associé à la sous-tangente en un point à la courbe d'équation $f(x)$ pour savoir si on a affairr à deux racines réclles ou à deux racines imaginaires.

\section{3 / L'utilisation de l'algorithme d'Euclide}

Ainsi les principes mis à part, Sturm ne reprend pas en pratique tous les raisonnements courants de l'analyse infinitésimale. Cela est d'autant moins nécessaire qu'il laisse tomber la suite des dérivées sur laquelle Fourier avait bâti son théorème, et lui substitue une suite construite par un procédé algébrique classique : l'algorithrne d'Euclide. Nous avons déjà dit que c'est là l'innovation majeure par rapport au théorème de Fourier. Innovation aussi par l'adaptation du procédé classique au problème résolu ; à chaque division le signe du reste est changé et ce changement, "inutile si l'on n'avait pour but que de trouver le plus grand commun diviseur, est nécessaire à (cette) théorie ". Nécessaire en effet pour produire la fameuse condition de définition d'une "suite de Sturm ". On voit bien, encore une fois, que Sturm procède artificiellement, c'est-à-dire invente.

L'algorithme d'Euclide modifié ne sert pas, dans le mémoire de 1835, à définir uno "suite de Sturm " en général. Pour cela, nous l'avons dit, il n'est besoin que d'énoncer les condilions nécessaires et suffisantes d'existence de talles suites. Il sert à construire explicitement une suite particulière, grâce à laquelle, d'ailleurs, Sturm démontre son théorème. Mais il joue un rôle certainernent décisif pour la postérité du théorème. Peut-être a-t-il suggéré à Sylvester ou Hermite, par exemple, la possibilité d'un recadrage théorique

(53) En particulier liv. I, \& 24. 
du théorème. Si, au lieu de tout baser sur les concepts de fonction et de continuité, on part du concept algébrique de polynôme, suite finie de nombres appelés coefficients, on peut, avec la construction algorithmique de la suite de Sturm, raisonner d'un bout à l'autre dans un cardre puremont algébrique, comme c'élait l'ambition de Lagrange que Fourier croyait irréalisable. Si, au lieu de considérer une seule équation à une seule inconnue, on envisage plus généralement des systèmes de $n$ équations à $n$ inconnues, alors un lien s'établit entre le théorème de Sturm et des résultats de la théorie de l'élimination algébrique, qui met sur la voie d'une déduction algébrique d'un résultat apparenté à celui de Sturm $\left(^{54}\right)$.

IV - LE: THÉOREME DE STURM GÉNÉRALISÉ PAR TARSKI

Ce n'est pourtant pas le résultat de Sylvester mais bien celui de Sturm qui attire, au début du $\mathrm{xx}^{\mathrm{e}}$ siècle, l'attention des mathématiciens engagés dans le développement de l'algèbre abstraite comme Artin et Schreier, ou des logiciens interpellés par le problème de la décision posé par Hilbert comme Tarski. L'artualité du théorème de Sturm est surtout liée à son utilisation par Tarski pour démontrer la complétude de l'algèbre et de la géométrie élémentaires ${ }^{55}$ ). 'Tarski en conçoit une vorsion encore plus générale que celle rendue familière par les travaux d'Hermite et de Sylvester $\left.{ }^{56}\right)$. Il envisage, en effet, des systèmes pouvant comprendre $p$ équations à $n$ inconnues (pour $n \neq p$ ) el $q$ inéquations $\dot{a} n$ inconnues. Conme il l'serit dans la note 12 de la deuxièm.

\footnotetext{
56 Nous laissons ici de cite cet aspect, aborde dans notre communicution au XVIle rongrès international d'llistoire des Sciences Berkeley, Californie, juilletaoût 198i).

(55) Ce résultat se trouve dans un mémoire dont on connalt deux versions, formellement assez différentes (voir n. 11 supra). Nous nous référons beaucoup ì la première, dont le texte commente de façon répétée la nature des services rendus par le théoreme de Sturm. Mais la seconde version est assortie d'une très longue note (n. 12) où Tarski reformule le théoreme en soulignant son lien a ses propres théorimes d'rlimination des quantificateurs.

68) Pour Sylvester se reporter a la n. 8. Pour llermite, voir Sur l'extension dn théorème de M. Sturm à un sýstème d'équations simultanées, dans les GEutres (Paris : Gauthier-Villars, 1905-1917), t. 1, 281-283 et III, 1-34. Et les Remarques sur le throrème de $\mathbf{M}$. Sturm, ibia., †. I, $184-287$.
} 
version de son mémoire, le théorème de Sturm fournit " un critère de résolubilité dans $\mathbf{R}$, qui implique aussi bien des inéquations quir des équations n.

\section{1 / Un critère "purement algébriqu" "}

Cie qui frapp dans le mémoire de Tarski eu égard au théorème de Sturm, c'est que celui-ci est présenté comme un théorème algébrique. Tarski fait remarquer que, pour les besoins de sa démonstration, "il est d'une importance fondamentale qu'il soit possible de construire pour la fonction donnée $f(x) \ldots$ les fonctions (de la chaine de Sturm) à l'aide do moyens purement algébriques "(57). C'est dire que Tarski est surtout intéressé, dans le théorème de Sturm, par l'emploi de l'algorithme d'Euclide ( $\left.{ }^{58}\right)$ qui permet de construire " une suite finie de fonctions rationnelles (de polynômes au sens algébrique) ". Les composantes non algébriques de la démonstration originale : le recours aux idées de variation infiniment petite ou de continuité, Tarski n'y prète aucune attention et les efface totalement, pour ainsi dire, de son interprétation du théorème de Sturm.

C'est que, d'une part, Tarski raisonne sur des polynômes et non sur des fonctions continues. Aussi n'a-t-il pas besoin de la version analylique du théorème des valeurs intermédiaires, mais seulement de ce qu'il en considère comme un " cas particulier ", celui où on en restreint l'application à des polynômes. La formulation de cet énoncé plus particulier se fait en termes algébriques qui décrivent des conditions de signe. C'est l'axiome XVII' de son système d'algèbre des nombres réels ( $\left.{ }^{59}\right)$.

D'autre part, étant donné l'usage qu'il veut faire du théorème

57) La complétude de l'algèbre el la géométrie démentaires, dans Logique, sémanlique, métamathémalique, 1923-1944 (Paris : Armand Colin, 1974), t. 2, p. 219.

(58) Pour Tarski, algorithme d'Euclide et théorème de Sturm sont liés de façon essentielle, en dépit de la possibiliti de construire des chatnes de Sturm par d'autres moyens. Dans l'introduction it la deuxième version de son mémoire, s'agissant de donner des exemples mathématiques connus de ce qu'on appellc une "méthode de décision ", il cite exclusivement l'algorithme d'Euclide (appliqué aux entiers naturels positifs et aux polynòmes a coefficients constunts; et le théorème de Sturm. Or, on peut penser encore it l'algorithme qui sert a calculer la racine carrie d'un nombre naturel (mais il s'agit d'un algorithme inflni, i.e. qui peut ètre indéfiniment continué).

(59) a La complétude... ", 1.12, p. 213-215. 
de Sturm : prouver son lemme d'élimination des quantificateurs ( $\left.{ }^{60}\right)$ pour la théorir élémentaire du corps ordonné des nombres réels ( $\left.{ }^{61}\right)$, il importe à Tarski de fournir un critère purement algébrique (62) de l'existence d'une solution réclle pour un système donné d'équations et d'inéquations polynomiales. L'existence d'un tel critère algébrique est en effet l'indice mathématique de l'existence d'un' procédure (métamathématique) d'élimination des quantificateurs. Le theorème de Sturm fournit bien, comme Tarski le souligne de façon répétée, ' type de critère. Cependant il s'agit évidemment pour Tarski, non du théorème dans sa facture originale, mais de "la méthode do. Sturm généralisée " $\left.{ }^{63}\right)$ qu'il a formulée el redémontrée dans le radre logico-algébrique qu'il a lui-même tracé. Tarski n'avait pas comme premier souci celui de l'exartitude historique! Mais Tarski infidèle, c'est Tarski réateur. Il fait un libre usage d'un theorème connu (64) et le transforme pour ainsi dirr spontanément, en sorte à l'adapter à ses propres buts (65). Sans entrer dans des détails dont la technicité nous entrainerait

\footnotetext{
6o: Il convienl de distinguer le lemme dobimination des quantiticateurs de lalgorithme correspondant. Si le premier est une trouvaille lumineuse, le second egl loin d'etre aussi simple en pratique sur une machine; qu'il l'est en théorie. Des travaux recents sont ainsi consacrés a simplifier l'algorithme de Tarski, c'est-i-dire it réduire le temps utile à un ordinateur pour son exécution.

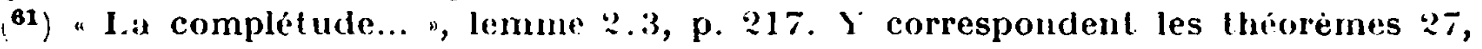
2:1, 31 de A Decision .Methud for Klemenlary Algebra and Germetry, 20 ed. Berkeley : University of California Press, 1951).

Tarski démontre que lout inonce existentiel de la forme $\exists$.r $A(x)$, ou $x$ représente un nombre réel quelconque el $A$ une conjonction d'equations et d'inéquations algébriques, est equivalent ì un énoncé ne comportant plus aucun quantificateur. Elémentaire se dit d'un énoncé ou d'un ensemble d'enoncés qui peuvent être formulés dans le langage logique du calcul des prédicats du premier ordre (les seules variables quantifiées itant des viriables d'individus:

(92) "Lal completude... ", p. 2lo' : l.e contenu mathématique du lemme 2.3 id'élimination des quantificateurs) se réduit ì ceci. Il est possible, en accord avec ce lemme, de fournir un critire :une condition nécessaire et suffisantej purement algébrique permettant de constater que toutes les íquations et inéquations (considéries) possèdent atu moins une solution relle commune iraciue . "Voir egalement le commentaire du lemme 2.5, p. $225-2: 26$.

(63) Ibid., 2.1, p. : $: 16$.

64) Tarski connat dabord le thioreme de sturm par les exposes de Il. Weber, de (.: Runge et de B. 1. Vin der Waerden. Ensuite, lat note $1:$ de A recision Wrhorl... permet de le penser, il a lu le mémoire original de l'auteur.

$\left.{ }^{65}\right)$ Il est intéressant de remarquer que Tarski et Sylvester, purtant tous deux de. la necessité d'un critère algébrique de la réalité des racines d'un polynòme, aboutissent, cia des intriêts fort difrirents, it des solutions relevint de points de rue totalement distincts.
} 
trop loin, disons que, dans le système de Tarski, un énoncé sans quantificateur est une combinaison (booléenne) de formules atomiques du type :

$$
\alpha=\beta \quad \text { ou } \quad \alpha>\beta,
$$

où $\alpha$ et $\beta$ sont des "termes " algébriques, c'est-à-dire des expressions logiquement bien formées à partir des éléments que sont les symboles de constantes ou de variables parcourant l'ensemble des nombres réels $(0,1, x, y, z, \ldots)$, et les symboles des opérations algébriques élémentaires, ce mot étant pris au sens usuel $:(+, \cdots, \times,:)\left({ }^{66}\right)$. Un énoncé sans quantificateur du système logique élémentaire (au sens de Tarski) pour le corps commutatif, ordonné, des nombres réels, c'est donc précisément une combinaison, ou, comme on dit habituellement, un système d'équations et d'inéquations algébriques. On voit le rapport, essentiel, entre algébrique tel que l'entend spontanément un mathématicien, et tel que l'entend aussi Tarski parlant de "critère purement algébrique ", et "énoncé sans quantificateur", notion logique forgée dans le contexte des solutions partielles au problème de la décidabilité $\left({ }^{67}\right)$. Généralisant le théorème de Sturm et rendant visible la procédure algébrique et la procédure logique impliquées dans cette généralisation, Tarski a montré que la théorie élémentaire du corps ordonné des nombres réels admet l'élimination des quantificateurs, c'est-à-dire équivaut à une théorie algébrique en un sens qui rejoint l'acception mathématique traditionnelle.

Celle-ci exig; pour le moins, en effet, l'rxclusion, dans les raisonnements, des notions de continuité ou d'infiniment petit, tt recoupe ainsi l'opposition banale Algèbre/Analyse ou théorie

(6) Turski ne se sert en fait que des symboles des trois operations d'addition, de soustraction et de multiplication. Cela implique une généralisation immédiate de ses résultats a la structure d'anneau unitaire ordonne. Insislons ici sur le fait que des symboles comme celui de l'exponentielle, du logarithme ou de l'integrale sont exclus de ce langage.

(87) Voir la liste de ces résultats jusqu'à 1951 dans l'introduction à $A$ becision Method for Elementary Algebra and Germetry, p. 12. Y ajouter celui de J. Herbrand, liecherches snr la thérsie de la démonstralion, ed. Jean Van Heijenoort (Paris : puf, 1968), chap. IV. Une théorie T, formulée dans un langage I., est. décidable s'il existe une procédure, dite de décision, permettant de décider dans chaque cas particulier si une proposition, écrite dans $\mathrm{J}$, peut ètre démontrée - se souvenir qu'une démonstration est une suite fnie de formules ! - par des moyens internes à $T$ (voir par exemple Tarski, A general method in proofs of undecidability, dans Undecidable Theories, en collab. avec Mostowski et R. M. Robinson (Amsterdam : North-Holland, 1953), p. 3). 
des équations/théoric des fonctions. Donner une théorie de $\mathbf{R}$, qui fût sinon algébrique du moins non analytique (ou non géométrique), a donc signifié à la fin du xixe siècle : trouver un ensemble d'axiomes, ne comportant parmi eux aucun axiome de continuité, et caractérisant néanmoins $R$, en ce sens que tout théorème vrai dans $\mathbf{R}$ soit déductible de cet ensemble. C'est ainsi que Drdekind a réussi à construire l'ensemble ordonné des nombres réels grâce à la méthode des coupures $\left({ }^{68}\right)$; que Hilbert, qui voulait échapper à une expression directe de la continuité, a dû cependant adjoindre aux axiomes de corps ordonné l'axiome d'Archimède et un "axiome d'inextensibilité " ( ${ }^{69}$ ) et que les mathématiciens de l'école algébrique américaine ont cherché quantité de varianles au système de Hilbert (70). Tarski connaissait evidemment tous res travaux (71). Lui-mème choisit tantôl l'axiome de Dedekind, comme dans son Introduclion à la logique ( $\left.{ }^{72}\right)$, tantôt le théorème de la borne inférieure : axiomr XVII du "système élémentaire d'algèbre des réels » con-truit dans "La complétude de l'algèbre et de la géométrie élémentaires ", lantôt et de préférence, une version algébriqur du theorème des valeurs intermédiaires : axiome XVII' du même système $\left.{ }^{73}\right)$. Cette préférence est par elle-même significative : il était bien connu que le contenu de l'axiome XVII' était au fondement d'une théorie des équations construite sans emprunt extérieur de concept ou de méthode.

Il est donc bien évident que Tarski veut construire un sys-

(8) Dedekind, sleliglieil und imalionale Zahlen, 1 re id. Braunschweig, 187:3; trad. Tranç. : La biblistheque d'Ornicar?:Diffusion Paris : I.e Seuil, 1978).

(69) Ilibert, Iber den Zahlbegriff, Jahresber. der Deutsch. Malh.-Iereinigung, $\therefore \quad 1900), 180-184$.

(70) Ces travaux, dus notamment a $\xi$. H. Moore, F. V. Huntington et O. Vellen, se trouvent dans les premiers volumes en particulier le V ot le VII des Transactions "f the American Mathematical Snciely.

71) Le chapitre $X$ de son Introdurlion à la logique (1 re éd. en polonais, 1936 ; trad. franc., Paris : Gauthier-Villars, 1960) intitule : "Fondements de l'arithmetique des nombres réels ", en témoigne. Voir en particulier la n. 1 de la p. 191 oì Tarski mentionne, is coté du système de libbert, celui, ou plutòt ceux de Iluntington (dont il recommande par ailleurs la monographie : The Fundamental Proposilions of Algebra, ed. by .J. W. A. Young (New York, 1911). Dans a Ia complétude de l'algèbre... "la note de la p. 23: renvoie au systeme de Huntington pour les complexes. Dans Les ensembles définissables de nombres réels (1931), traduit dans I.ogique, sémantique, mélamalhematiqur, t. I, il renvoie à un systène de o. Velien.

(72) Chap. $X$, trad. frans., p. 191-198.

i3) Cet axiome esf celui conserve par la version de 1948 : A Derision Methri..., נ1. 9. p. 48-49. 
tème algébrique des nombres réels $\left({ }^{74}\right)$. Or il apportre trois résultals nouveaux qui sont, au fond, des déterminations concrètes lu projet algébrique d'ensemble :

1 / S'apercevant que tous les axiomes de continuité sont logiquement complexes puisqu'ils exigent l'usagre de variables désignant des ensembles de nombres réels, il montre néanmoins qu'on peut caractériser dans le langage du calcul des prédicats du premier ordre la théoric algébrique des nombres réels. D'où le qualitatif "élémentaire " pour son système (infini puisque XVII" wt un schéma d'axiome).

2 / En explicitant au préalable la collection des symboles primitifs du langage utilisé, il met en évidence le matériau nécessaire : deux symboles pour les constantes d'individus 0 ot 1 ; des symboles de variables d'individus ( ${ }^{75}$ ) ; trois symboles pour les opérations d'addition, de soustraction et de multiplication; enfin deux symboles pour les relations d'égalité ct d'inégalité. Rien que de très élémentaire en somme (au sens banal du terme), mais qui suffit cependant à construire l'objet " polynòme à coefficients réels ", et à ramener la question méta mathématique de la décidabilité de la théoric élémentaire de $\mathbf{R}$ à celle de la résolubilité dans $\mathbf{R}$ d'un système d'équations et d'inéquations.

3 / La " méthode de Sturm généralisée " prrmet de montrer" que la théorie algébrique élémentaire $\left.{ }^{76}\right)$ de $\mathbf{R}$ admot l'élimination

\footnotetext{
74) Nous laissons ici de cotte la construction algébrique des corps réels d'ArtinSchreier, publice en 1927, mais dont. Tarski ne tient explicitement compte ru'en 194s: A Decision Melhod..., n. 9 et 15.

L'axiomatique d'Artin-Schreier est celle qui est en usage aujourd'hui. Nous en avons analysé ailleurs la signification et la portée (Communication au Colloque d'Ilistoire des Mathématiques, crnu, Marseille-Iuminy, juin 1983); voir aussi l'ouvrage (a parattre) : Corps et modeles. Aspects de la construrlion de l'algibre réelle, $I^{\text {e }}$ partie.

(76) Ces variables prennent leurs valeurs dans le corps des nombres réels ou, comme le remarque ensuite Tarski, dans le corps des nombres réels algébriques. C'est pourquoi il s'agit bien d'une théorie algébrique de $\mathbf{R}$.

${ }^{\left({ }^{76}\right)}$ C'est à dessein que nous parlons de théorie algébrique el clémentaire, de même que Tarski écrit : "système élémentaire d'algèbre des récls". Aujourd'hui, compte tenu de l'observation que les structures de l'algèbre abstraite : groupe, anneau, corps, module, etc., et leurs différentes spécifications comme, par exemple, groupe abélien divisible, corps algébriquement clos, anneau ordonné, etc., peuvent être axiomatisées (de Paçon finie ou dénombrable) dans le langage logique du calcul des prédicats du premier ordre, certains logiciens tendent à considérer qu'une theorie est caracterisable algébriquement si elle est caractérisable dans le premier ordre. C'est par exemple, la convention adoptée par Kreisel-Krivine, Elements de logique mathèmatique, Mono-
} 
des quantificateurs et qu'elle est, par conséquent, décidable. Compte tenu de $1 /$ et 2 /, on comprend l'importance qu'accordait Tarski à ce que le théorème de Sturm permit d'avoir un " critère purement algébrique " de la réalité des racines d'un polynôme ( $\left.{ }^{77}\right)$.

Nous venons de montrer que la question dr. la nature, algébrique ou non, du théorème de Sturm se pose surtout depuis l'existence de la méthode d'élimination des quantificateurs de Tarski (qui est la "méthode de Sturm généralisée "). Indice d'une discussion encore actuelle sur l'utilisation de méthodes de démonstration non algébriques pour des propositions exprimables algébriquement, elle correspond aussi à un intérêt métamathématique: aigu pour la signification du terme "algébrique ". Cet intérêt est thématisé notamment dans certains travaux de Tarski ( ${ }^{78}$ ) ot d'A. Robinson (19) que nous ne pouvons examiner iei.

\section{2 / Le premier exemple d'élimination des quantificateurs pour le cas réel}

Cie qu'a fait Tarski du théorème de Sturm justifie largement la "sensation " produite à sa naissance. Tous ces caractères quo lui reconnaissaient, par une vue plus ou moins claire, les mathématiciens, le logicien less a confirmés : simplicité, effectivité algorithmique (de principe), importance fondamentale pour les mathématiques.

graphie de la smF : Paris : Dunod, 1967 ; chap. 3, resume, p. 33, et appendice 1 , p. 1:in Ifais cette convention est loin d'ètre acceptée d'une facon générale.

Signalons quen tout état de cause une theorie algébrique n'admet pas forcément. l'élimination des quantiflcateurs. On a ainsi montré récemment qu'un anneau orronné n'a cette propriété que s'il verifie les axiomes de corps ordonné et réel-clos.

$\left.{ }^{77}\right)$ Jans la deuxième version de son travail, Tarski insistera plutòt sur le fait que le theoreme de Sturm fournit une condition elémentaire (du premier ordre) de l'existence d'une racine réelle pour un polynòme donné Voir A Hecision Wethod..., n. 1?, p. $\overline{1} 1,2 e$ al.

78) Citons comme un bon representant de ce vecteur dans les recherches de Tarski : Fquational I.ogic and equational theories of algebra, dans Coll. Log. Follnd. Malh. llannover, 1966, publié en 1968 (Amsterdam : North-Ilolland)), 275-288, qui reprend certaines idées exprimées par J. C. C. Mckinsey dans The decision problem for some :lasses of sentences without quantifiers, Journal of symbolic Jogir, 8 (1943).

(29) Pour A. Robinson, une "algèbre d'axiomes "est un ensemble d'axiomes, qui comporte un symbole de relation pour l'égalité. Une structure est algébrique si elle est axiomatisée par une algèbre d'axiomes voir par exemple On the Metamalhemalics of Ilgebra (Amsterdam: North-Holland, I951), chap. VI). Robinson definit par ailleurs la notion métamathématique de prédicat formulej algébrique par analogie avec crlle d'élément algébrique sur un corps ibid., chap. VIII). 
Que d'auteurs ont été éblouis par la simplicité de notre théoreme ( $\left.{ }^{80}\right)$ ! Tarski a montré d'une faģon précise qu'il se laisse 'n fait algébriser sans problème et sans stcours extéricur (81) ; et qu'il s'écrit dans un langage logique du premier ordre. Bref, tout commr le systeme construit par Tarski pour les nombres récls, ot dans lequel on peut l'exprimer, il est algébrique of élémentaire.

Tarski a encore confirme l'intention, diversement mais très fréquemment formulée dès 1829 , qu'il s'agissait d'un théorèmo rapital. Il a, en fait, déterniné la raison de cotte importance : I. théorème de Sturm permet de faire, pour li cas du corps ordonné $\mathbf{R}$ (muni, outre de la relation $=$, de la relation $>$ ), une théorie parallèle à celle dı: l'élimination algébrique classique. - On appelle ainsi l'ensemblo des méthodes de résolution des systèmes d'équations algébriques à plusieurs inconnues (systèmes qui, du point de vur logique, sont écrits aver la seule relation $=$ ) par élimination successive des inconnues. - Il apparaîtra, "nsuite, plus clairement que cette théorie peut ètre, dans certains cas, avantageusement substituér à celle de l'élimination algébrique ou à d'autres méthodes algébriques particulières, sa généralité faisant d'elle une méthode simple et d'une grande clarté conceptuelle. A. Robinson en a ainsi déduit une preuve triviale du dix-septième problème de Hilbert ( $\left.{ }^{82}\right)$.

Tarski, enfin, a tiré toul lo parti théorique du caractère effectif du théorème. La tradition logique, représentée par G. Boole of par E. Schröder, d'une algèbre de la logique, c'est-à-dire d'une: théorie logique à l'image de la théorir des équations, fut à l'origine: d. l'idée que l'élimination des quantificateurs d'une formule logique rendait soluble pour cette formule le problème de la décision. C'est ainsi qu'on trouve dans un mémoire de L. Löwenheim (1915), grandement influencé par les travaux et le langage de Schröder, la première esquisse de cette méthode de décision appli-

80; Nous avons cité plus haut Terquem (référence dans la n. 3). Mais nous pourrions evoquer bien d'autres èloges, de liouville, de Borchardt, de Serret, de Hermite, etc. Fetenons peut-être que Hermite avait salué dans ce théorème "un exemple rare de simpliciti: et d'élégance ", qui " ouvre lère nouvelle de l'Algèbre moderne "lettre i Borchardt du 28 janvier 18:4, dans (Eunres, 1 . I).

(81) Tout en êtant moins général que al'Algèbre réelle "d'Artin-Schreier, le système ilémentaire d'algèbre des réels de Tarski appréhende cependant le théorème de Sturm d'une façon analogue $\mathrm{et}$ fort éloignie de l'approche également algébrique de Sylvester ou d'Ilermite).

$\left.{ }^{82}\right)$ On ordered fields and definite functions, Mathematische Annalen, 130 (1955): 
quéc au calcul monarlique (83) des prédicats du premirer ordre. D'autre part, lorsque Hibert et Arkermann formulent en 1928 (84) le problème général de la décision. le paradigme de la theorie des équations est bien entendu toujours présent, mais un accent partirulier est mis sur l'aspect de calculabilité effective. Pour eux, an effet, l'usage du symbolisme logique pour l'exposé formel des théories (axiomatiques en particulier) laisse espérer la mise au point d'un traitement permetlant de décider, systématiquement (c'est-à-dire pour une formule quelconque) et an un nombre fini l'étapes, si telle formule donnée est valide dans telle théorie. En Lermes plus modernes, on dirait que c'èt ait définir par là l'idée d'un algorithme de décision ( ${ }^{85}$ ).

Or. Tarski remarque (en 1948) que les mathematiques traditionnelles offrent au moins deux exemples de tels algorithnes. Celui d'Euclide fournit, en effet, une procédure de décision pour la classe des propositions vraies du type " $p$ et $q$ sont premiers entw. rux ", où $p$ et $q$ sont des nombres entiers ou des polynômes à coulficients constants. Celui de Sturm permet de décider la vérité de: propositions de la forme "le polynôme $p$ a exactement $n$ racines distinctes dans l'intervallo (A, B) \#. Par construction, mais ausi par nature, le second est plus complexe que le premier, car les propositions décidées par lui nécessitent normalement pour leur formulation logique l'usage de quantificateurs. Si l'on explicite, on trouve une formule du genre:

$$
\begin{aligned}
\exists x_{1} \exists x_{2} \ldots \exists & x_{n}\left\{\left[\left(x_{1}<x_{2}\right) \wedge\left(x_{2}<x_{3}\right) \wedge \ldots \wedge\left(x_{n-1}<x_{n}\right)\right.\right. \\
& \wedge\left(\mathrm{A} \leqslant x_{1}\right) \wedge\left(x_{n} \leqslant \mathrm{~B}\right) \\
& \wedge\left(p\left(x_{1}\right)=0\right) \wedge\left(p\left(x_{2}\right)=0\right) \wedge \ldots \wedge\left(p\left(x_{n}\right)=(1)\right] \\
& \Rightarrow \forall y((\mathrm{~A} \leqslant y \leqslant \mathrm{~B}) \wedge(p(y)=0)) \\
& \left.\left.\Rightarrow\left(\left(y=x_{1}\right) \vee\left(y=x_{2}\right) \vee \ldots \vee\left(y=x_{n}\right)\right)\right]\right\}\left({ }^{86}\right) .
\end{aligned}
$$

83) Monadique signife qu'on se restreint aux predicats ì une place, ce qui implique 'juantiflcation non multiple.

84) Grundzüge der theorelischen Jugik Berlin : Springer-Verlate, 1938:, \$ 11-1:, 1. $72-81$.

$\left.{ }^{85}\right)$ Voir par exemple la défnition des concepts de décidabilité et de calculabilité dans II. Ilermes, Aufzählbarkeit, Entscheidbarkeil, Bererhenbarkeit Berlin : SpringerVerlag, 1961), chap. 1 .

Un ensemble d'éléments $E$ est décidable si et seulement si sa fonction caractéristique $\chi_{E}$ est calculable. Une fonction est calculable s'il existe un algorithme, c'est-n-dire une procédure de calcul effectif, pour calculer la valeur de la fonction pour n'importe quelle valeur de son argument.

(88) $\times \mathrm{A} \leqslant x_{1}$ " est une abriviation pour: "A $\mathrm{A} x_{1}^{\prime} \vee \mathrm{A}=r_{1}^{\prime}$ ". 
Cependant, Tarski montre que, grâce au théorème de Sturm, cette proposition est équivalente à une proposition sans quantifi-

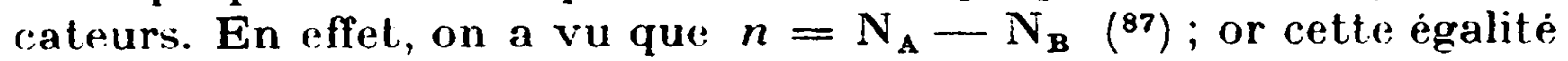
peut ètre exprimée par une combinaison booléenne d'égalités at d'inégalités polynomiales dont les termes sont obtenus rationnellement à partir des coefficients de $p$ et des bornes de l'intervalle. Par exemple, dans le cas d'un polynôme du second degré $x^{2}+b x+c$ la suile de Sturm est $\left(x^{2}+b x+c, 2 x+b, \frac{b^{2}}{4}-c\right) ; \mathrm{N}_{\mathrm{A}}-\mathrm{N}_{\mathrm{B}}=2$ (en supposant qu'on est dans of cas) s'exprime par la conjonction $\left(^{88}\right)$ :

$$
\begin{aligned}
\left(b^{2}-4 c>0\right) \wedge\left(\mathrm{A}^{2}\right. & +\mathrm{A} b+c>0) \wedge(2 \mathrm{~A}+b>0 \\
& \wedge\left(\mathrm{B}^{2}+b \mathrm{~B}+c>0\right) \wedge(2 \mathrm{~B}+b>0) .
\end{aligned}
$$

Dans le ras où l'intervalle n'est pas spécifié, on retrouve la condition bien connue : $b^{2}-4 c>0$.

Ainsi, le théorème de Sturm est un magnifique exemple d'élimination (implicite) dés quantificateurs. C'est même le premier exemple non trivial : on ne sait pas a priori si un polynôme a une racine réelle dans un intervalle donné, alors que si on se place, par exemple, dans lo corps des complexes on sera sûr de toute façon dr l'existence de racines en nombre égal au degré du polynôme.

Sturm aurait sans doute été bien surpris de la mélamorphose de son théorème. L'historien l'est, à vrai dire, à peine moins. Ne voilà-t-il pas, en effet, un résultat de l'Analyse, dont la rigueur ot le progrès furent étroitement liés, à la fin du siècle dernier, à la théorie de la quantification multiple (G. Frege), qui se voit ensuite transformé en prototype de méthode d'élimination des quantificateurs? On dira pour le moins que les voies de l'histoire sont parfois bien indirectes!...

CNRS, Paris.

Hourya Benis-Sinaceur.

(87) Supra, ler paragraphe, p. 102.

(88) Ce cas volontairement choisi pour sa simplicité ne donne cependant pas une dée exacte de la longueur de l'algorithme pour un polynome de degré plus élevé. 